\title{
Genetic characterization of maize germplasm derived from Suwan population and temperate resources
}

Xun Wu, Angui Wang, Xiangyang Guo, Pengfei Liu, Yunfang Zhu, Xiushi Li and Zehui Chen*

\begin{abstract}
Background: The Suwan population is a well-known maize germplasm that has greatly contributed to the development of maize breeding in tropical and subtropical regions, especially in southern China. Inbred lines derived from the Suwan population always contain stronger resistance and extensive adaptability in different environments. To evaluate the genetic character of inbred lines derived from the Suwan population, a panel including 226 inbred line derived from the Suwan population and temperate resources was assembled and genotyped by using MaizeSNP50 BeadChip, which contained 56,110 genome-wide single nucleotide polymorphism (SNP) markers. This panel contained 98 temperate inbred line and 128 lines derived from the Suwan population.

Results: The results showed that high genetic diversity was found, with PIC and GD to be 0.67 and 0.60 , respectively. In addition, two novel subgroups were identified, with representative inbred lines as HCL645 and Ki32, respectively. One acknowledged heterotic group of lowa Stiff Stalk Synthetic (SS) was also identified in this study. This study can provide some additional scientific evidence for heterotic group division and use in maize. Additionally, lower linkage disequilibrium (LD) levels and weaker genetic relationships were found, with an average LD level of $41.15 \mathrm{~kb}$ that varied from 3.5 to $96 \mathrm{~kb}$. A total of $82.8 \%$ of paired relative kinships ranged from 0.05 to 0.28 .
\end{abstract}

Conclusions: These results would not only facilitate maize breeding practices in tropical and subtropical regions, but also revealed that this panel can be used in dissecting the genetic basis of complex quantitative traits' variations by using genome-wide association studies (GWAS).

Keywords: Maize germplasm, Genetic characterization, Suwan population

\section{Introduction}

Maize (Zea mays L.) rapidly spread throughout the world after its domestication from teosinte in tropical Mexico [14]. Many local landraces or farmer's varieties were formed owing to the repeated introduction, artificial selection, and adaptation to different environments. Some representative inbred lines with different origins were produced, and relevant hybrids were released. To continuously improve the performance of hybrids adapting to partial ecological environments effectively, heterotic groups and heterotic patterns were clarified [11, 12, 26], which played important roles in maize commercial breeding programs. However, to date, less than $5 \%$ of

\footnotetext{
* Correspondence: chenzh907@sina.com

Institute of Upland Food Crops, Guizhou Academy of Agricultural Sciences, Guiyang 550000, Guizhou, China
}

germplasm is used in maize breeding around the world, and less than $1 \%$ is used in the U.S. [4].

A few classical inbred lines were repeated used to develop new lines and excellent hybrids in temperature conditions, which contributed mostly to maize hybrid breeding [20]. i.e., B73, PH207, Mo17 [13], and Huangzaosi (HZS) [26].For instance, most lines belonged to Stiff Stalk (BSSS) and were derived from B73, and approximately $35 \%$ of Pioneer's hybrids were from BSSS. Mo17 was common used in all U.S. seed companies, and more than $45 \%$ of Pioneer's commercial hybrids contain the pedigree of Mo17 [19]. In the Huang-Huai River region of China, HZS is widely used during the maize breeding process, has derived more than 40 elite lines and 80 hybrids and has greatly contributed to the industrial development of Chinese maize. For example, the maize hybrid 'Zhendan 958' (Zheng58 $\times$ Chang7-2) has 
been grown in an area of more than 35 million ha in China [25].

In addition, Tuxpeño is one of the well-known maize germplasms that has greatly contributed to the development of tropical maize breeding programs [1]. Tuxpeño maize pools often contain a broad genetic base and show larger diversity than that in temperate populations. Tuxpeño germplasm's productivity and crossing it with the race ETO result in Tuxpeño-ETO heterotic patterns in tropical maize breeding, which are widely used in Thailand, Colombia, and Mexico [23]. However, during the Tuxpeño maize pools' ultilization process, its lower resistance to some diseases and insect pests in tropical regions was found, which limited its widely use. Therefore, some improvements were done during maize breeding practice. Since the 1960s, 36 accessions have been collected across the world, and relevant agronomic traits have been evaluated in order to improve maize adaptation under Thailand's cultivation conditions. Based on the results of data analysis, one synthetic variety of Suwan1 with excellent performance was released through modified ear-to-row selection methods, which showed better performance in tropical regions [21], and rapidly spread to tropical and subtropical regions as a result. The heterotic pattern by crossing lines from Suwan1to temperate resources became famous and was widely used in subtropical regions, especially in southwest China. However, knowledge on the genetic differences between Suwan population and temperate maize germplasm is still limited, which remains one of the impediments to its increased use in breeding programs.

For decades, many marker-based studies have addressed genetic diversity, population structures, and genetic relationships by using different panels and markers Wang et al. [24] showed that only four heterotic groups were identified when integrating only the Chinese elite lines from temperature regions together, which was smaller than that found by using tropical and temperate lines together [11, 28]. In Yan's studies, 632 lines from temperate and tropical/subtropical public breeding practices were used: it contained 351 tropical/subtropical lines from the Maize and Wheat Improvement Center (CIMMYT) and showed much higher genetic diversity than that in the temperate panel [11]. However, fewer markers and resources of maize germplasm were used in other reports, which would provide limited information when dissecting the potential genetic diversity in maize germplasm.

By using the genotyping by sequencing (GBS) method, Romay et al. [17] evaluated the genetic characters of 2815 accessions in the USA national maize inbred seed bank. Based on 681,257 SNP markers, many more rare alleles were identified, but only a modest amount of the available diversity was found in the commercial germplasm. Strong population stratification was identified, with a small number of key lines as the center of one cluster. This genotypic information provided abundant insight into maize genetic diversity around the world. However, most lines used in previous studies were publicly available, representing historical recombinants during the maize domestication and improvement progress. Along with the development of private commercial maize breeding, more and more new elite inbred lines were produced and widely used in modern maize breeding practice. This was especially true for the Suwan population, which came from tropical regions, and derived many more elite lines widely used in tropical/subtropical maize breeding program, such as Ki32, QR273, and QB446. Therefore, collecting one naturally diverse panel that contained the latest lines derived from temperate and Suwan resources that are widely used in modern breeding programs is better for in-depth study of the genetic character of maize germplasm. Based on this panel, we dissect the genetic relationship between pair-lines by using the high-throughput genotyping method, which is important not only for expanding gene pools to accelerate maize breeding progress but also for providing additional information for evaluating whether this panel is suitable for genome-wide study (GWAS) in analyzing variation of complex traits.

In this study, 226 accessions derived from the Suwan population and temperate resources were selected and genotyped by using the MaizeSNP50 BeadChip, including approximately 56,110 single nucleotide polymorphism (SNP) markers. The objectives were to evaluate the genetic diversity, population stratification, relative kinship, linkage disequilibrium (LD) level, and whether this panel is suitable for GWAS of agronomic traits' variation.

\section{Results}

\section{Model-based population structure and relative kinship}

The most significant peak in $\Delta K$ was observed (Fig. 1a) when $\mathrm{k}$ was three. This suggests that this panel of maize germplasm could be divided into three groups (Fig. 1b), with representative lines of HCL645, Ki32, and B73, respectively. These results were consistent with the experimental breeding according to the pedigrees and knowledge of breeding history. Twenty-seven lines were included in the HCL645 group, with most lines derived from HCL645. Seventy-eight lines were contained in the Ki32 group, with many lines derived from Ki32, or accessions introduced from tropical regions. The B73 group contained 120 lines, mainly being derived from B73 and its descendants. Furthermore, when $\mathrm{k}$ is six, the second peak of $\Delta K$ was observed, meaning that this panel could be further divided into six subgroups, with representative lines of HCL645, Ki32, QR273, Mo17, A801, and B73, respectively (Fig. 1a). The 

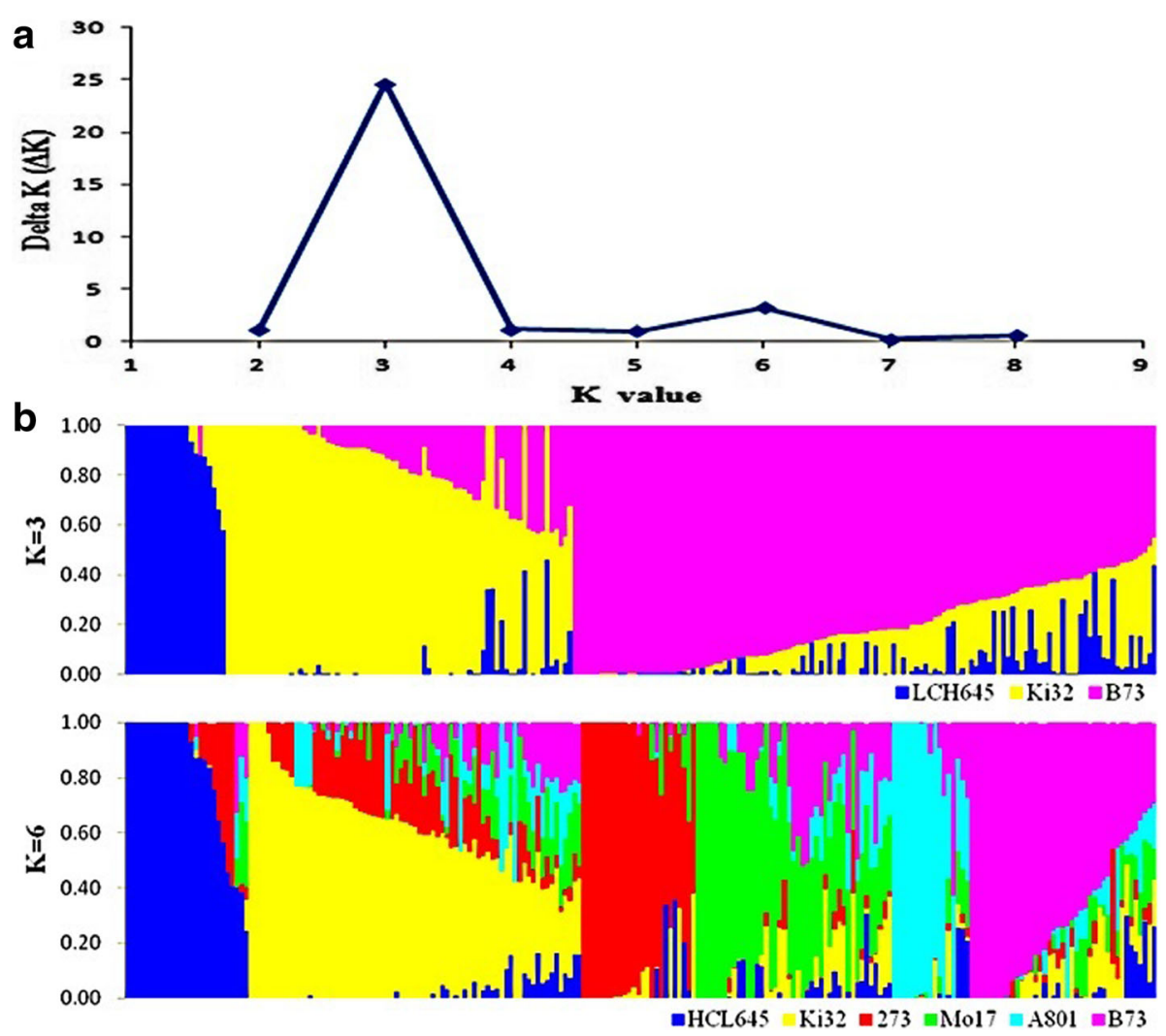

Fig. 1 Model-based population structure division. Delta $K$ was calculated from $k=2$ to $k=8$ (a). $\mathbf{b}$ showed the population structure division of the 226 accessions by using 8905 SNPs

other lines with membership probabilities $<0.5$ were appointed into one cluster called the mix group.

Estimation of relative kinships showed that, in this panel: $82.8 \%$ of paired relative kinship ranged from 0.05 to $0.28,0.33 \%$ of paired relative kinship equaled to 0 , $0.38 \%$ of paired relative kinship ranged from 0 to 0.05 , and the remaining ranged from 0.30 to 0.80 (Fig. 2). This analysis indicated that a weak or various relative kinship existed in this collection of elite maize germplasm, which was consistent with known pedigree/sources of the 226 lines.

\section{Neighbor-jointing tree and principal component analysis}

A neighbor-joining tree was constructed based on the modified Euclidean distance and is shown in Fig. 3. The 226 accessions were clustered into six major groups according to their origins, with representative lines of CLH645, CML171, B73, HZS, Qi319, and Mo17 (Fig. 3). The Huangzaosi (HZS) cluster contained 87 inbred lines, with lots derived or included Suwan genetic material in their pedigrees. The B73 cluster contained 29 inbred lines, the Mo17 cluster contained 44 lines, the HCL645 cluster contained 27 lines, the CML171 cluster contained 13 lines, and the Qi319 cluster contained 17 lines. These results showed moderately common ground with some difference compared to that evaluated by using model-based method. The principal component analysis (PCA) results showed obvious population differences among HCL645, Iowa Stiff Stalk Synthetic (SS), tropicalderived lines, P groups, and Non-Stiff Stalk (NSS) subgroups, with representative lines including HCL645, B73, Ki32, P178, and Mo17. Lines derived from hybrid offspring of the Suwan (SW) population crossing with NSS population were pointed into tropical-derived subgroup, with smaller distance to NSS lines when comparing with that between Suwan and NSS lines. Lines derived from hybrid offspring of the Tuxpeño population crossing with the Reid population were distributed in the middle of tropical-derived population and the SS population (Fig. 4).

\section{Linkage disequilibrium (LD) level and genetic diversity}

Linkage disequilibrium evaluation results showed that the average LD level is $41.15 \mathrm{~kb}$ in the panel of 226 lines and varies from 3.5 to $96 \mathrm{~kb}$ across 10 chromosomes (Chr.), with $3.5 \mathrm{~kb}$ on Chr.1, and $96 \mathrm{~kb}$ on Chr.5, Chr.6 and Chr.10 (Table 1; Fig. 5). For the whole panel of 226 lines, a total of 293,060 alleles were detected. The average gene diversity was 0.67 , varying from 0.50 to 0.84 , and the average PIC was 0.60 , varying from 0.38 to 0.82 . 


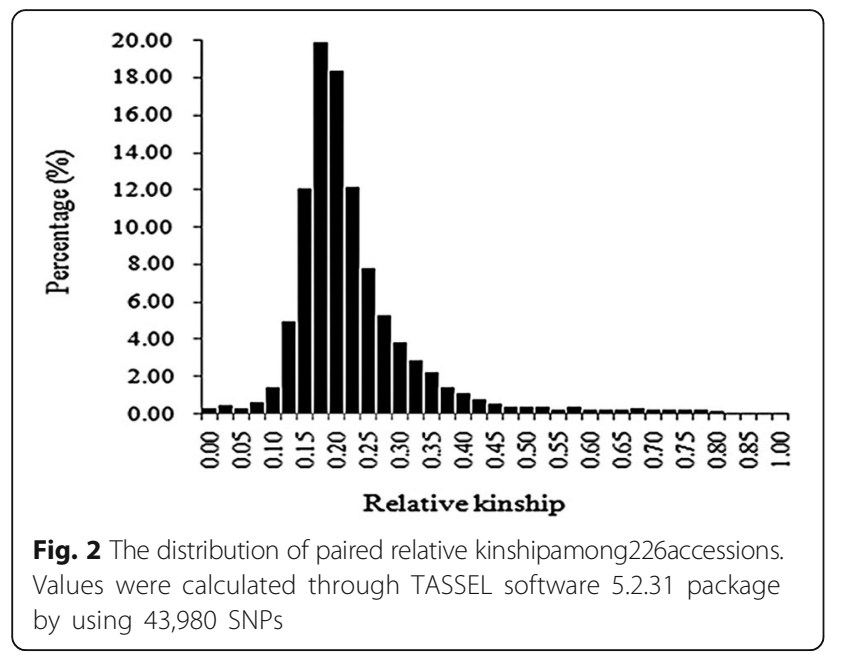

\section{Discussion}

Specific subpopulation structure division was found in this panel

Population structure analysis is usually a primary task in maize breeding research. When using multiple panels with different genetic backgrounds, some inconsistent results were reported. In China, four main heterotic groups are always clarified, with representative inbred lines of Huangzaosi, Ye478, Mo17, Lv28, and P178 [24, 26]. In the United States, NSS and SS are two mainly heterotic groups that play important roles in maize breeding both historically and currently (van [22]). When joining tropical and temperate maize accessions into one panel, two subgroups are always divided first, and then much more complex population structure differentiation information is revealed in-depth [11, 26, 27]. In this study, three subgroups are

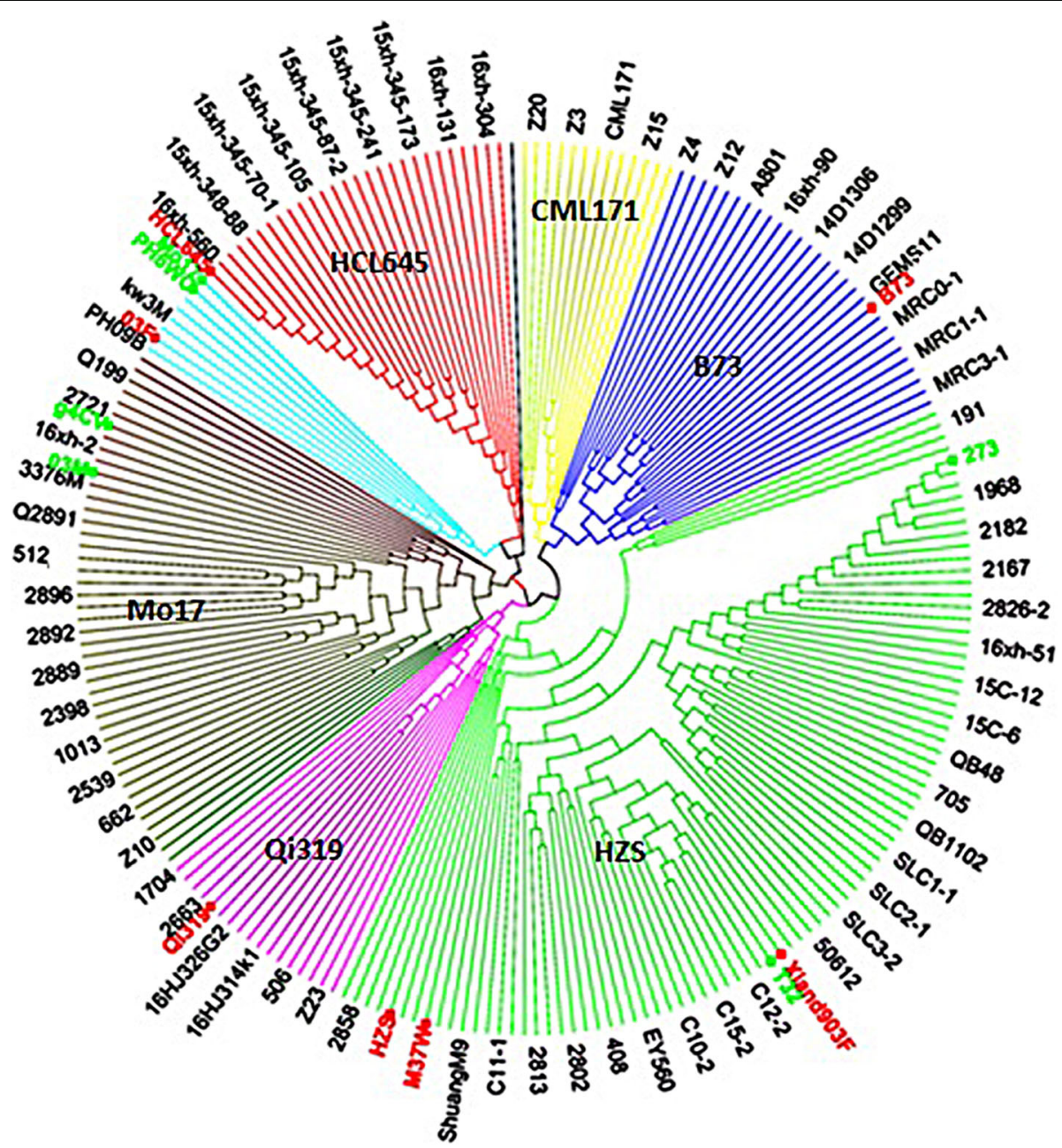

Fig. 3 Neighbor-joining trees of the 226 maize accessions. Mo17 is a representative inbred line of Non-Stiff Stalk (NSS). Qi319 is a representative inbred of the Ludahonggu group. 'HZS' is an abbreviation of Huangzaosi, which is a representative inbred of the Tangsipingtou group (TSPT). B73 is a representative inbred of Stiff Stalk Synthetic (SS). CML171 is one representative inbred of tropical/subtropical group. In addition, HCL645 is one representative inbred of Monsanto 


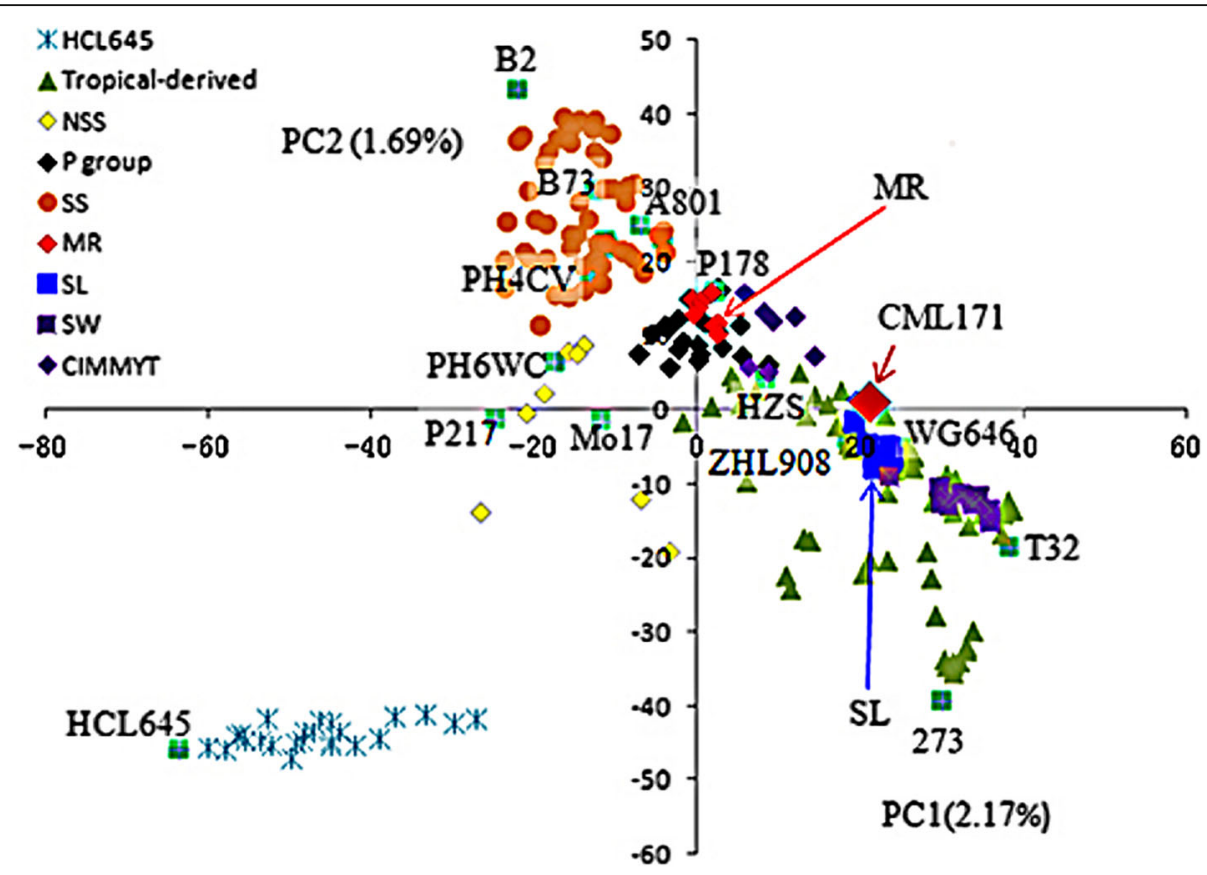

Fig. 4 Results of principal components (PCs). 'HCL645' is one representative line of Monsanto accessions. 'Tropical-derived' means accessions containing tropical genes in their pedigrees. 'NSS' means Non-Stiff Stalk (NSS) subgroup. 'SS' means lowa Stiff Stalk Synthetic group. 'MR' means accessions derived from the generations of Tuxpeño crossing with SS germplasm. 'SL' means accessions derived from the generations of Suwan crossing with Lancaster germplasm. 'CIMMYT' means accessions come from Maize and Wheat Improvement Center (CIMMYT)

divided first, with representative lines selected as HCL645, Ki32, and B73, respectively (Fig. 1). Most lines belonging to the subgroup Ki32 were selected directly from the landrace of the Suwan population, or contained Suwan blood in their pedigrees. For instance, an inbred line of Ki32 is selected from the landrace of Suwan1 population, directly. QB446 was selected from the crossers of the Suwan1 population and Mo17. The

Table 1 Average LD level of the 10 chromosomes for $r 2$ greater than 0.1

\begin{tabular}{ll}
\hline Chromosome & $\begin{array}{l}\text { Linkage disequilibrium } \\
(\mathrm{LD})(\mathrm{Kb})\end{array}$ \\
\hline 1 & 24 \\
2 & 6 \\
3 & 3.5 \\
4 & 6 \\
5 & 96 \\
6 & 96 \\
7 & 48 \\
8 & 12 \\
9 & 24 \\
10 & 96 \\
Average & 41.15 \\
\hline
\end{tabular}

two lines were also appointed into the same subgroup of Ki32. The heterotic mode of crossing lines from the subgroup of B73 with lines from the subgroup of Ki32 is widely used for maize breeding in the southern Chinese environment. Some excellent hybrids were released and widely used for maize production in southern China, such as Jinyu838 with parental lines QB446 and QB1018, wherein QB1018 belongs to the subgroup of B73 and QB446 to the subgroup of Ki32. In addition, one novel subgroup of HCL645 was identified, which contained many lines with HCL645 in their pedigrees. Dika517 is one important hybrid with HCL645 in one parental line: it was one example of a heterotic mode crossing lines belonging to the subgroup of HCL645 with lines belonging to the subgroup of B73. Dika517 was widely cultivated in the northern parts of China. However, lines from the subgroup HCL645 also showed higher combined abilities when crossing with lines from the subgroup of Ki32. For instance, crosses derived from 15xh-345-59 and 50,612 are compared in regional maize testing for the second time, and showed better performance in tropical or subtropical environments. 15xh-345-59 belongs to the subgroup of HCL645, and 50,612 belong to the subgroup of Ki32. These results showed abundant proof of maize population structure differentiation, which provides new insight for maize germplasm improvement in tropical or subtropical regions. 


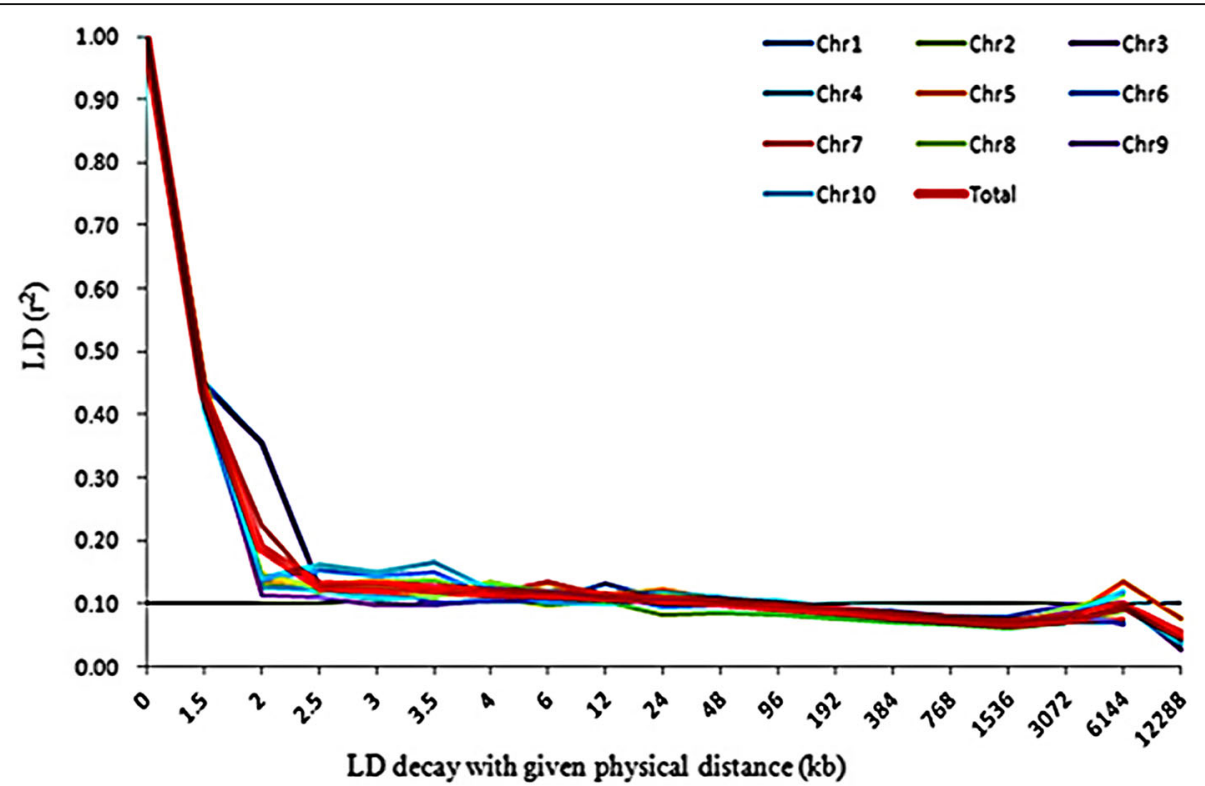

Fig. 5 Linkage disequilibrium (LD) level. 'Chr1-Chr10' means chromosome 1 to 10. 'Total' means the whole genome

\section{Abundant genetic diversity existed in this panel}

Knowledge of genetic diversity among accessions is indispensable in maize research and breeding programs, which provide scientific proof for the evaluation of detection resolution for specific panels and quantitative traits [8]. For one panel, significantly more genetic diversity would show stronger population structure differentiation, lower LD levels, and weaker pair-relationships. Research showed that different population compositions would impact genetic diversity. Tropical maize germplasm always showed much more genetic diversity, lower LD levels, and weaker pair-relationships [28]. In this study, lower LD levels were found, with average LD decay distance to be $41.15 \mathrm{~kb}$, varying from 3.5 to $96 \mathrm{~kb}$ (Table 1 and Fig. 5). This was much smaller than that when using elite temperate maize germplasm only, with average LD decay distance at $328 \mathrm{~kb}$, varying from 272 to $595 \mathrm{~kb}$ [26]. However, the LD levels found in this study were also larger than that identified in Yan's report, with average LD levels at 5-10 kb, varying from 1 to $10 \mathrm{~kb}$ [28]. One possible reason is that in Yan's panel, many original tropical accessions were included, and much more genetic diversity existed. However, in this study, where many improved tropical lines were derived from tropical populations and some local landraces, the tropical blood ratio was lower. In addition, gene diversity (GD) of 0.67 and PIC of 0.60 was identified in this study, which was obviously higher than that reported before, with GD and PIC ay 0.36 and 0.29 [26]. One possible reason for this phenomenon is that much more tropical maize germplasm were included in this study. In our previous research, 367 elite lines were collected. Because of the narrower genetic base among lines, stronger pair-relationships existed, with $94.97 \%$ of paired relative kinship ranging from 0.05 to 0.28 [26], which was significantly higher than that detected in this study, with $82.8 \%$ of paired relative kinship varying from 0.1 to 0.4 (Fig. 2). Because of this, we integrated maize germplasm from Suwan and temperate regions together in this study, wherein the Suwan population showed much more abundant genetic diversity than that of temperate populations $[12,27]$. These results showed that the panel used in this study contained more abundant genetic diversity, along with lower LD levels, which would provide higher detection resolution for using genome-wide association study to dissect the variation in complex quantitative traits in maize.

\section{Conclusions}

In this study, we clarified the population structure differentiation of maize germplasm derived from Suwan germplasm and related improved lines. Two novel subgroups were found, with representative lines identified as Ki32 and HCL645, respectively. However, much more genetic diversity and lower LD level were also detected, which revealed that this panel could be used for the dissection of quantitative traits' genetic variation by using the genome-wide association study (GWAS) strategy. These results would provide some additional information for heterotic group division and use. As a result, this may facilitate better maize breeding, especially in tropical and subtropical regions. 


\section{Materials and methods}

\section{Plant material}

The maize panel used in this study contained 226 accessions derived from the Suwan population and temperate resources. These contained 98 temperate accessions, with representative lines of B73 and Mo17, which were widely used in maize breeding historically [10]. In addition, most temperate lines were used in the latest maize breeding program in China, such as QB1013 and HC3. In addition, 128 accessions derived from the Suwan population were also selected, such as Ki32, QR273, and QB446, all of which are widely used in maize breeding practices in the southwest of China. Detailed information is listed in Additional file 1: Table S1.

\section{Genotyping and quality evaluation}

This panel was genotyped by using MaizeSNP50 BeadChip, including 56,110 SNPs. Those SNPs were evenly distributed across the maize genome based on the B73 reference sequence (www.illumina.com/maizeSNP50). Leaves were collected from the five-leaf stage of maize seedlings. The tip of young and tender maize leaves of five plants were collected as a bulk to extract genomic DNA, according to the modified CTAB procedure [18]. Then, 200 microgram $(\mu \mathrm{g})$ genomic DNA of each sample was selected to do further analysis. DNA quality assessment and genotyping work was done at the Beijing Compass Biotechnology Company, according to the Infinium ${ }^{\oplus}$ HD assay ultra-protocol guide. A total of 54,976 of 56,110 SNPs were called successfully among the 226 lines. SNPs with a missing rate of more than $20 \%$ and minor allele numbers (MAF) of $<0.05$ were excluded from the genotyping dataset. Then, the source sequences of remaining SNPs were identified through BlastN searches against the reference genome sequence of B73 (RefGen-V1, http://www.maizegdb.org/). SNPs with ambiguous physical positions or multiple blast-hits were excluded from the genotyped dataset. After that, 43,980 high-quality SNPs were used in further analysis.

\section{Model-based population structure analysis}

In total, 8905 SNPs with high genetic diversity, low missing rates, and even distribution across the genome were selected to investigate the population structure differentiation of the 226 lines, by using the modelbased approach [24]. Structure V2.3.3 software [6] was run with $\mathrm{k}$ (the number of populations) from 1 to 15 , with five runs for each $k$, with a burn-in period of 10,000 and 10,000 replications. The ad hoc statistic delta $K(\Delta K)$ was used to determine the number of clusters [3]. Outputs of Structure were integrated by using CLUMPP software [7]. Lines with membership probabilities of more than 0.5 were assigned to corresponding clusters when incorporation with pedigree information for each lines [26].
Construction of neighbor-joining tree and principal component analysis (PCA).

To obtain an in-depth picture of the genetic relationships among 226 inbred lines, the genetic distance between pair-lines was calculated by using 43,980 SNPs. Modified Euclidean was defined as follows: D $=1$ - identity by state (IBS) similarity [8]. IBS means the probability that alleles derived at random from two individuals at identical loci are the same. For any two lines, the probability of IBS was averaged over all non-missing loci. Then, the cladogram was constructed based on the distance matrix described above, by using the neighborjoining (NJ) algorithm [30]. Clusters were observed from the result of the phylogenetic tree. All calculations were carried out in TASSEL software 5.2.31 [2]. PCA of 226 lines was done according to the Patterson's description [15] using TASSEL software 5.2.31 [2].

\section{Linkage disequilibrium (LD) evaluation}

By using 43,980 SNPs with MAF > 0.05 and missing data < $20 \%$, pair-SNPs' LD level was evaluated using the squared Pearson correlation coefficient $\left(r^{2}\right)$ between vectors of SNP alleles according to the method described by Hill [5]. The cutoff value of $\mathrm{r}^{2}$ was set to be 0.1 [28]. TASSEL 5.2.31 software was run with a $20 \mathrm{~kb}$ slide-window size [7], the spacing between two loci on the same chromosome was segmented in distance bins of $50 \mathrm{~kb}$, and the average LD was assessed for each bin [16].

\section{Summary statistic of 226 lines}

The pair relationship among the 226 inbred lines was estimated by definition as follows: $F_{i j}=\left(Q_{i j}-Q_{m} /\left(1-Q_{m}\right)\right.$, where $Q_{i j}$ is the probability of IBS for random loci from $i$ and $j$, and $Q_{m}$ is the average probability of IBS for loci from random individuals. Pair-kinship coefficients of 0,0 to 0.1 , and 0.1 to 0.5 indicated weak, intermediate, and strong similarity between accessions, respectively [29]. The gene diversity (GD) and polymorphism information content (PIC) were calculated using PowerMarker V3.25 [9], with GD as the probability that two alleles randomly chosen from the test sample were different [11]. The PIC was calculated as follows: $\widehat{\mathrm{PIC}}_{1}$ $=1-\sum_{\mathrm{u}=1}^{\mathrm{k}} \tilde{\mathrm{P}}_{\mathrm{lu}}^{2}-\sum_{\mathrm{u}=1}^{\mathrm{k}-1} \sum_{\mathrm{v}=\mathrm{u}+1}^{\mathrm{k}} 2 \tilde{\mathrm{P}}_{\mathrm{lu}}^{2} \tilde{\mathrm{P}}_{\mathrm{lv}}^{2}$, where $p l u$ and $p l v$ are the frequencies of the $u$ th and vth alleles for marker 1 , respectively.

\section{Additional file}

Additional file 1: Table S1. Summary information of the 226 accessions. (XLS $25 \mathrm{~kb}$ )

\section{Abbreviations}

$\triangle K$ : delta K: BSSS: Stiff stalk; Chr: Chromosomes; CIMMYT: Maize and Wheat Improvement Center; GBS: Genotyping by sequencing; GD: Gene diversities; 
GWAS: Genome-wide association study; HZS: Huangzaosi; IBS: Identity by state; LD: Linkage disequilibrium; MAF: Minor allele numbers; NSS: Non-Stiff Stalk; PCA: Principal component analysis; PIC: Polymorphism information contents; SNP: Single-nucleotide polymorphisms; SS: lowa Stiff Stalk Synthetic; SW: Suwan

\section{Acknowledgements}

During the research process, some works were completed by Minlun Yang. This paper was reviewed by some academic reviewers, lots of good suggestions were raised for the improvement of this study. Thanks for all their help.

\section{Funding}

This research was supported by the National Key Research and Development Program of China (2016YFD010120206-4), the National Natural Science Foundation (31760387), the Guizhou Academy of Agricultural Science Innovation Program ([2014] 006), the Guizhou Natural Science Foundation ([2017]1413), Guizhou Major Special Projects ([2013]6022), Guizhou Science and technology support program ([2017]2507, [2018]2296, and [2017] 2504-1). And Qiankehe platform talent ([2018] 5629).

\section{Availability of data and materials}

All data and material supporting our findings were presented within the manuscript or additional supporting files.

\section{Authors' contributions}

XW and ZC conceived the study and conducted the design of experiment. $X W, A W$ and $X G$ implemented the experiment data analysis, and XW wrote the manuscript. All authors read and approved the final manuscript.

\section{Ethics approval and consent to participate}

Not applicable.

\section{Consent to publication}

The authors all have read and agreed to its content and are accountable for all aspects of the accuracy and integrity of the manuscript in accordance with ICMJE criteria. They all consent this manuscript to publish.

\section{Competing interests}

The authors declare that they have no competing interests.

\section{Publisher's Note}

Springer Nature remains neutral with regard to jurisdictional claims in published maps and institutional affiliations.

Received: 3 September 2018 Accepted: 14 December 2018 Published online: 10 January 2019

\section{References}

1. Amiruzzaman M, Islam MA, Pixley KV, Rohman MM. Heterosis and combining ability of CIMMYT's tropical late white maize. Int J Sustain Agric. 2011;3(3):76-81.

2. Bradbury PJ, Zhang Z, Kroon DE, Casstevens TM, Ramdoss Y, Buckler ES. TASSEL: software for association mapping of complex traits in diverse samples. Bioinformatics. 2007;23(19):2633-5.

3. Evanno G, Regnaut S, Goudet J. Detecting the number of clusters of individuals using the software STRUCTURE: a simulation study. Mol Ecol. 2005;14(8):2611-20.

4. David H, Khairallah M, Reeves T, Ribaut J-M, Skovmand B, Taba S, et al. Plant genetic resources what can they contribute toward increased crop productivity? Proc Natl Acad Sci U S A. 1999;96:5937-43.

5. Hill WG, Weir BS. Maximum-likelihood estimation of gene location by linkage disequilibrium. Am J Hum Genet. 1994;54(4):705-14.

6. Hubisz MJ, Falush D, Stephens M, Pritchard JK. Inferring weak population structure with the assistance of sample group information. Mol Ecol Resour. 2009;9(5):1322-32.

7. Jakobsson M, Rosenberg NA. CLUMPP: a cluster matching and permutation program for dealing with label switching and multimodality in analysis of population structure. Bioinformatics. 2007;23(14):1801-6.

8. Legesse BW, Myburg AA, Pixley KV, Botha AM. Genetic diversity of African maize inbred lines revealed by SSR markers. Hereditas. 2007;144(1):10-7.
9. Liu K, Muse SV. PowerMarker: an integrated analysis environment for genetic marker analysis. Bioinformatics. 2005;21(9):2128-9.

10. Lorenz A, Hoegemeyer T. The phylogenetic relationships of US maize germplasm. Nat Genet. 2013:45(8):844-5.

11. Lu Y, Yan J, Guimaraes CT, Taba S, Hao Z, Gao S, et al. Molecular characterization of global maize breeding germplasm based on genomewide single nucleotide polymorphisms. Theor Appl Genet. 2009;120(1):93115.

12. Lukens L, Wen W, Franco J, Chavez-Tovar VH, Yan J, Taba S. Genetic characterization of a Core set of a tropical maize race Tuxpeño for further use in maize improvement. PLoS One. 2012;7(3):e32626.

13. Mikel MA, Dudley JW. Evolution of north American dent corn from public to proprietary germplasm. Crop Sci. 2006;46(3):1193.

14. Mir C, Zerjal T, Combes V, Dumas F, Madur D, Bedoya C, et al. Out of America: tracing the genetic footprints of the global diffusion of maize. Theor Appl Genet. 2013;126(11):2671-82.

15. Patterson N, Price AL, Reich D. Population structure and eigenanalysis. PLoS Genet. 2006;2(12):e190

16. Riedelsheimer C, Czedik-Eysenberg A, Grieder C, Lisec J, Technow F, Sulpice $\mathrm{R}$, et al. Genomic and metabolic prediction of complex heterotic traits in hybrid maize. Nat Genet. 2012;44(2):217-20.

17. Romay MC, Millard MJ, Glaubitz JC, Peiffer JA, Swarts KL, Casstevens TM, et al. Comprehensive genotyping of the USA national maize inbred seed bank. Genome Biol. 2013;14(6).

18. Saghai-Maroof MA, Soliman KM, Jorgensen RA, Allard RW. Ribosomal DNA spacer-length polymorphisms in barley: mendelian inheritance, chromosomal location, and population dynamics. Proc Natl Acad Sci U S A. 1984;81(24):8014-8.

19. Smith JSC, Duvick DN, Smith OS, Cooper M, Feng LZ. Changes in pedigree backgrounds of pioneer brand maize hybrids widely grown from 1930 to 1999. Crop Sci. 2004:44(6):1935-46.

20. Smith S. Pedigree background changes in US hybrid maize between 1980 and 2004. Crop Sci. 2007:47(5):1914-26.

21. Timothy DH, Harvey PH, Dowswell CR. Develepment and spread of improved maize varieties and hybrids in developing countries. Bureau for Science and Technology Agency for International Development Washington, DC 1988:1-25.

22. van Heerwaarden J, Hufford MB, Ross-Ibarra J. Historical genomics of north American maize. Proc Natl Acad Sci U S A. 2012;109(31):12420-5.

23. Vasal SK, Srinivasan G, Crossa J, Beck DL. Heterosis and combining ability of CIMMYT's tropical late white maize germplasm [in Thailand, Colombia and Mexico]. Crop Sci. 1992;32(4):884-90.

24. Wang R, Yu Y, Zhao J, Shi Y, Song Y, Wang T, et al. Population structure and linkage disequilibrium of a mini core set of maize inbred lines in China. Theor Appl Genet. 2008;117(7):1141-53.

25. Weng J, Xie C, Hao Z, Wang J, Liu C, Li M, et al. Genome-wide association study identifies candidate genes that affect plant height in Chinese elite maize (Zea mays L.) inbred lines. PLoS One. 2011:6(12):e29229.

26. Wu X, Li Y, Shi Y, Song Y, Wang T, Huang Y, et al. Fine genetic characterization of elite maize germplasm using high-throughput SNP genotyping. Theor Appl Genet. 2014;127(3):621-31.

27. Wu X, Li Y, Li X, Li C, Shi Y, Song Y, et al. Analysis of genetic differentiation and genomic variation to reveal potential regions of importance during maize improvement. BMC plant biology. 2015;15:256.

28. Yan J, Shah T, Warburton ML, Buckler ES, McMullen MD, Crouch J. Genetic characterization and linkage disequilibrium estimation of a global maize collection using SNP markers. PLoS One. 2009:4(12):e8451.

29. Yang X, Xu Y, Shah T, Li H, Han Z, Li J, et al. Comparison of SSRs and SNPS in assessment of genetic relatedness in maize. Genetica. 2011:139(8):1045-54.

30. Zhang W, Sun Z. Random local neighbor joining: a new method for reconstructing phylogenetic trees. Mol Phylogenet Evol. 2008:47(1):117-28. 